\title{
Failures of Categoricity and Compositionality for Intuitionistic Disjunction*
}

\author{
Jack Woods
}

July 10, 2013

This is the penultimate draft of a paper forthcoming in Thought: A Journal of Philosophy. Please cite the published version.

There are at least two reasonable types of logical inferentialism. The first type of inferentialism views the meaning of a connective in proof-theoretic terms, having no truck with ordinary model theory except in its role as an instrument to illuminate various proof-theoretic features of a connective. ${ }^{1}$ The second type of inferentialism is more modest, allowing that we have some prior conception of the meaning that the natural deduction rules for a particular connective determine. $^{2}$ Such a position would look to the conditions forced by acceptance of a set of rules against a background model theory for an account of connective meaning. This position is moderate, plausible, and problematic. In the case of classical logic, against the presumption of standard extensional semantics, Carnap long ago demonstrated the general failure of proof-rules to induce the intended boolean interpretation of the connectives. ${ }^{3}$ There have been a number of attempts to solve this problem by modifying the natural deduction format, but none have achieved anything like a consensus. ${ }^{4}$

Recent work by James Garson has shown that if we instead modify the model-theoretic assumptions, we can generate meanings for many connectives

\footnotetext{
*Thanks to John Burgess, Lloyd Humberstone, Jimmy Martin, the audience at the Arché Foundations of Logical Consequence closing project, and especially to James Garson for useful discussion.

${ }^{1}$ Perhaps most famously exposited in Prawitz (1985).

${ }^{2} \mathrm{~A}$ version of this approach is argued for in Peacocke (1976). From here on, I use 'meaning' for the familiar sort of model-theoretic "truth"-conditions for connectives. I make no distinction between the sense and reference of a connective here as such distinctions would distract instead of illuminate.

${ }^{3}$ See (Carnap, 1959, 89-94). There is a way-exploited in Peacocke (1976) — to induce the boolean interpretation of the connectives from the classical proof-rules, but it is too coarse, inducing the boolean interpretation of the connectives from the intuitionistic natural deduction rules. This is clearly not in the spirit of the modest inferentialist program.

${ }^{4}$ Notable versions of this strategy include a move to multiple-conclusion versions of natural deduction(Shoesmith and Smiley (1978), the introduction of primitive signs of rejection Smiley (1996), and a synthesis of both approaches recently defended in Restall (2005).
} 
and sets thereof which are categorical in the sense of uniquely extending an assignment of semantic values to the atomic sentences of a language. Garson's results are most impressive when we restrict ourselves to the intuitionistic propositional calculus. He shows that when we generalize our semantics to allow intensional truth-conditions by viewing a model as a set of assignments of $\mathbf{T}$ and $\mathbf{F}$ to formulas of a language, we recover familiar intuitionistic interpretations of negation $(\neg)$, conjunction $(\&)$, and the conditional $(\rightarrow)$. Moreover, as is to be expected, these interpretations are semantically separable in the sense that each interpretation conservatively extends the others. ${ }^{5}$ For example, no purely implicational formula is ratified by the set of models satisfying the conditions for negation and implication that wasn't already ratified by the set of models satisfying merely the implicational condition.

This is a welcome advance for the modest form of inferentialism. Given an interpretation of these general models, we can use Garson's method to generate meanings for the connectives in terms of this interpretation. For example, if we think of an assignments of $\mathbf{T}$ and $\mathbf{F}$ as representing stages of verification as in the typical presentation of intuitionistic semantics in terms of Kripke models, we can use Garson's results to derive standard intuitionistic interpretations of the connectives from the natural deduction rules for the intuitionistic propositional calculus. Let a modest inferentialist be someone who holds that the meanings of the connectives are generated by Garson's method over suitably interpreted general models by their single-conclusion natural deduction rules. ${ }^{6}$ A minimal commitment of being a modest inferentialist in this sense is the conviction that general models can be interpreted in a plausible fashion which does justice to their prior conception of the meaning of the connectives.

For intuitionists, this is a relatively easy commitment to fulfill as there is an entirely standard interpretation of intuitionistic logic which fits general models. For classical logicians, it is not as straightforward. The condition expressed by classical rules such as double negation elimination has the unwelcome effect of barring certain assignments of $\mathbf{T}$ and $\mathbf{F}$ to the atomic sentences of the language. This is a very unwelcome consequence; a modest inferentialist should require that the meanings of the connectives be conservative over the basic states of affairs represented by assignments of $\mathbf{T}$ and $\mathbf{F}$ to atomic sentences. A plausible classical interpretation of general models needs to accommodate this fact. Work in this direction has been done by both Garson and Ian Rumfitt

\footnotetext{
${ }^{5}$ In Garson (2001), he suggests that this gives the guts of an argument that the intuitionistic meanings of the connectives are in some sense more properly logical. In more recent work (Garson (forthcoming)), he does not adopt such a position. The results below can be taken to add to Garson's reasons for backing off this assumption as the undesirable properties of the meaning of $\vee$ when generated as in Garson (2001) conflict with intuitions about the correct behavior of logical operators.

${ }^{6}$ We will thus ignore inferentialists who opt for multiple-conclusion formulations of natural deduction, those who presume that the meaning generated by a set of natural deduction rules must be extensional, and bilateralists who formulate natural deduction rules in terms of both acceptance and rejection.
} 
who show that it is possible to characterize the condition expressed by double negation elimination as a condition holding for all sentences. ${ }^{7}$ In conjunction with an interpretation of general models as representing a space of possibilities or, really, partial specifications of possible worlds, it is possible to argue that this condition is part of the pre-logical semantic framework presumed by the modest inferentialist instead of a condition expressed by the rules governing negation or the conditional. This type of position seems the best recourse for the classical modest inferentialist though we will see below that it is not entirely unproblematic.

The news is not all good for the modest inferentialist. The meaning generated by Garson's method for disjunction has a pair of undesirable properties. It is not categorical in the sense above of not uniquely extending an assignment of semantic values to the atomic sentences of a language and it is not weakly compositional in a sense to be spelled out below. The remainder of this note will establish these facts by constructing (a) an assignment of semantic values to the atomic sentences of a language which does not uniquely extend to an assignment of semantic values to all disjunctions, and (b) a pair of assignments which essentially disagree only on the semantic value of some atomic sentence, yet which assign differing semantic values to a disjunction of which this sentence is not a subformula. I will also discuss the upshot of these results for more definite interpretations of the models over which the condition is defined. The upshot of my discussion is that the failures of categoricity and completeness for disjunction is a serious problem which needs to be addressed by anyone adopting a modest inferentialist position though, admittedly, it is a cleaner problem for the intuitionistically inclined. Our discussion will thus be focused on the intuitionistic case. We now turn to the details.

Let $A t$ be a countable set of atomic sentences. Given an n-ary connective \#, let the language $\mathfrak{L}_{\#}$ be the closure of $A t$ under the formula generating operation $\#\left(A_{1}, \ldots, A_{n}\right)$. Given a language $L$, let a valuation be a function from $L$ to $\{\mathbf{T}, \mathbf{F}\}$. Call a function from $A t$ to $\{\mathbf{T}, \mathbf{F}\}$ an atomic valuation. A sequent is an ordered pair consisting of a set of formulas $\left\{A_{0}, \ldots, A_{n}\right\}$ called premises, and a formula $C$ called the conclusion. Natural deduction rules are similarly sets of ordered pairs consisting of a set of premise sequents and a conclusion sequent. For convenience, we will often write a rule $R$ in a vertical form like so:

$$
\text { (MP) } \frac{\Gamma \vdash \phi \quad \Gamma \vdash \phi \rightarrow \psi}{\Gamma \vdash \psi} \quad(\mathrm{CP}) \frac{\Gamma, \phi \vdash \psi}{\Gamma \vdash \phi \rightarrow \psi}
$$

Writing $v(\Gamma)=\mathbf{T}$ for $v(\psi)=\mathbf{T}$ for every $\psi \in \Gamma$, we will say that a valuation $v$ respects a sequent $\Gamma \vdash \phi$ if and only if $v(\Gamma)=\mathbf{T} \Rightarrow v(\phi)=\mathbf{T}$. Since we are concerned with intuitionistic logic and since intuitionistic semantics are intensional,

\footnotetext{
${ }^{7}$ Both Garson (forthcoming) and Rumfitt (2012) draw on ideas initially developed in Humberstone (1981).
} 
we need a generalized notion of a valuation. Let a general model of a language $L$ be an ordered pair $\left\langle W, V^{A}\right\rangle$ where $W$ is a non-empty set and $V^{A}$ a function from $W$ to the set of valuations of $L$. Let an atomic submodel be a pair $\left\langle W, V^{A} *\right\rangle$ where $V^{A} *$ is a function from $W$ to the set of atomic valuations of $L$. For most of what we do here, we can simplify considerably and think of a general model $A$ as a set of valuations $V=\left\{V_{v}^{A}: v \in W\right\} .{ }^{8}$ Given a general model $V$, we say that a rule respects $V$ if and only the conclusion sequent is respected by each $v \in V$ if every premise sequent is respected by every $v \in V$. So, for example, MP is respected by $V$ just in case $\Gamma \vdash \phi \rightarrow \psi$ and $\Gamma \vdash \phi$ are respected by every $v \in V$ only if $\Gamma \vdash \psi$ is similarly respected. To make this slightly easier, we will say that a sequent is correct on a set of valuations $V$ if and only if it is respected by every $v \in V .{ }^{9}$ We say a rule $R$ preserves correctness on a set of valuations $V$ if and only if every $\left\langle\left\{A_{0}, \ldots, A_{n}\right\}, C\right\rangle$ in $R$ is such that each of $A_{0}, \ldots, A_{n}$ is correct on $V$ only if $C$ is correct on $V$.

The natural way to extract model-theoretic meaning from a set of rules is to generate the set of models which, in some sense, respect these rules. In the ideal case, the set of models will admit of a description in terms of the truth-behavior of complex formulas in terms of the truth-behavior of their subformulas. Given a set of rules $R^{*}$, we can generate the family of sets of valuations $\mathfrak{V}_{R^{*}}$ containing each set of valuations $V$ for which the rules in $R^{*}$ preserve correctness on $V$. We state without proof two nice features of $\mathfrak{V}_{R^{*}}: 10$

1. A rule $\left\langle A_{0}, \ldots, A_{n}, C\right\rangle$ preserves correctness on every $V$ in $\mathfrak{V}_{R^{*}}$ if and only if we can derive $C$ from $A_{0}, \ldots A_{n}$ using $R^{*}$.

2. A sequent $\Gamma \vdash \phi$ is correct on $V$ for every $V \in \mathfrak{V}_{R^{*}}$ if and only if $\Gamma \vdash \phi$ is derivable from $R^{*}$.

Characterizing the model-theoretic meaning of a connective \# generated by $R^{\#}$ by the behavior of \# throughout $\mathfrak{V}_{R^{*}}$ allows a fine-grained measure of the model-theoretic meaning. For example, this method generates distinct modeltheoretic meanings for the set of rules comprised of MP and CP (henceforth abbreviated $R^{\rightarrow}$ ) and that of the set of rules comprised of MP, CP, and Peirce's Law.

As Garson demonstrates in Garson (2001), we can characterize $\mathfrak{V}_{R \rightarrow}$ by a condition on $V \in \mathfrak{V}_{R \rightarrow}$. We define a relation $\leq$ on $V$ as follows. Let $v \leq v^{\prime}$ iff $\forall \phi v(\phi)=\mathbf{T} \Rightarrow v^{\prime}(\phi)=\mathbf{T}$. A set of valuations $V$ ordered under $\leq$ is a familiar structure, resembling the notion of a Kripke model for intuitionistic logic. ${ }^{11}$ The

\footnotetext{
${ }^{8}$ We revert to the more complicated description of a model to ease our discussion of compositionality below. At all other points, we simply talk about sets of valuations. I will also customarily drop 'general' to ease readability.

${ }^{9}$ Garson (2001) calls this property $V$ - validity, but I find the term 'correctness' more suggestive.

${ }^{10}$ See (Humberstone, 1996, 457-458) for more details and proof. Our usage of derivable here presumes the standard structural rules of cut, weakening, and reflexivity.

${ }^{11}$ See Kripke (1965).
} 
condition for $\rightarrow$ is likewise familiar:

$\left(C^{\rightarrow}\right) \forall v \in V, v(\phi \rightarrow \psi)=\mathbf{T}$ iff $\forall v^{\prime} \in V,\left[v \leq v^{\prime} \& v^{\prime}(\phi)=\mathbf{T}\right] \Rightarrow v^{\prime}(\psi)=\mathbf{T} .{ }^{12}$

It is easily seen that some $V$ satisfy this condition. We now show that $\left\{V: V\right.$ satisfies $\left.C^{\rightarrow}\right\}=\mathfrak{V}_{R \rightarrow}{ }^{13}$

$p f$. Left-to-Right is trivial. Right-to-Left: Given $V$ for which $R^{\rightarrow}$ preserves correctness, $\phi, \psi \in \mathfrak{L}_{\rightarrow}$, and $v \in V$, we show $v(\phi \rightarrow \psi)=\mathbf{T}$ iff $\forall v^{\prime}\left(\left[v \leq v^{\prime}\right.\right.$ and $\left.\left.v^{\prime}(\phi)=\mathbf{T}\right] \Rightarrow v^{\prime}(\psi)=\mathbf{T}\right)$. Assume $v(\phi \rightarrow \psi)=$ $\mathbf{T}$. Given $v^{\prime} \in V$ such that $v \leq v^{\prime}$ and $v^{\prime}(\phi)=\mathbf{T}$, let $\Gamma=\{\phi, \phi \rightarrow$ $\psi\}$. Since $v \leq v^{\prime}, v^{\prime}(\phi \rightarrow \psi)=\mathbf{T}$. (MP) preserves correctness on $V$, so $\Gamma \vdash \psi$. Since $v^{\prime}(\phi)=v^{\prime}(\phi \rightarrow \psi)=\mathbf{T}, v^{\prime}(\psi)=\mathbf{T}$. Conversely, assume $v(\phi \rightarrow \psi)=\mathbf{F}$. Let $\Gamma_{v}$ be the set of formulas which hold at $v$. Since $v(\phi \rightarrow \psi)=\mathbf{F}, \Gamma_{v} \nvdash \phi \rightarrow \psi$. So, from the fact that (CP) preserves correctness in $V$, we get that $\Gamma_{v}, \phi \nvdash \psi$, so there is a $u \in V$ such that $u\left(\Gamma_{v}\right)=\mathbf{T}, u(\phi)=\mathbf{T}$ and $u(\psi)=\mathbf{F}$. Since $u\left(\Gamma_{v}\right)=\mathbf{T}$, $v \leq u$.

The only difference between the account of the conditional in Kripke semantics and the above account is that the Kripke account makes use of a different partial order on models defined only on At: $v \leq^{*} v^{\prime}$ iff $\forall p_{\in A} v(p)=\mathbf{T} \Rightarrow v^{\prime}(p)=\mathbf{T}$. However, it can easily be seen that the above condition is equivalent to the Kripke condition as the full partial order $\leq$ can be uniquely generated from the partial order $\leq^{*}$. Similar results can be obtained for the rules governing intuitionistic negation, and conjunction. ${ }^{14} V \in \mathfrak{V}_{R^{\vee}}$ can also be characterized, but the truth-condition for $\vee$ has a number of surprising features.

The natural deduction rules for $\vee$ are as follows:

$$
\begin{array}{cc}
(\vee \mathrm{I}-\mathrm{R}) \frac{\Gamma \vdash \phi}{\Gamma \vdash \phi \vee \psi} & (\vee \mathrm{I}-\mathrm{L}) \frac{\Gamma \vdash \phi}{\Gamma \vdash \psi \vee \phi} \\
(\mathrm{CD}) \frac{\Gamma \vdash \phi \vee \psi}{\Gamma, \phi \vdash \rho} & \Gamma, \psi \vdash \rho \\
\hline
\end{array}
$$

To characterize $\mathfrak{V}_{R^{\vee}}$ we assume $\leq$ is defined as above. We give an equivalent formulation of Garson's condition for $\vee:^{15}$

$$
\begin{aligned}
& C^{\vee}: v(\phi \vee \psi)=\mathbf{T} \text { iff } \forall \chi v(\chi)=\mathbf{F} \Rightarrow \exists v^{\prime}\left(v \leq v^{\prime} \text { and } v^{\prime}(\chi)=\mathbf{F}\right. \text { and } \\
& \left.\left[v^{\prime}(\phi)=\mathbf{T} \text { or } v^{\prime}(\psi)=\mathbf{T}\right]\right) .{ }^{16}
\end{aligned}
$$

\footnotetext{
${ }^{12}$ This is a slight change from Garson (2001) to fix a scoping error.

${ }^{13}$ This proof is due in its essentials to Garson (2001).

${ }^{14}$ Obtaining the correct intuitionistic condition for negation requires that we exclude the valuation which assigns every formula $\mathbf{T}$.

${ }^{15}$ The equivalent form is found in Humberstone (1996) (459).

${ }^{16}$ It is important to note that unlike $C^{\rightarrow}, \leq$ as it appears in this condition cannot be replaced with $\leq *$. See (Garson, 2001, 126).
} 
Now we show that $\left\{V: V\right.$ satisfies $\left.C^{\vee}\right\}=\mathfrak{V}_{R^{\vee}}$.

$p f$. Left-to-Right: Suppose $V$ satisfies $C^{\vee}$. Further suppose $\Gamma \vdash \psi$ holds throughout $V$. Let $v(\Gamma)=\mathbf{T}$. So, $v(\phi)=\mathbf{T}$. We need to show that for any $\chi \in \mathfrak{L}_{\vee}$ such that $v(\chi)=\mathbf{F}$ there is a $v^{\prime}$ extending $v$ such that $v^{\prime}(\chi)=\mathbf{F}$ and either $v^{\prime}(\phi)=\mathbf{T}$ or $v^{\prime}(\psi)=\mathbf{T}$. Since $v \leq v$ and $v(\phi)=\mathbf{T}$, we can take $v=v^{\prime}$. Similarly, the other two rules preserve correctness. Right-to-Left: Given $V$ for which $R^{\vee}$ preserves correctness and $v \in V$ such that $v(\phi \vee \psi)=\mathbf{T}$, let $\Gamma_{v}$ be set of formulas which hold at $v$. It follows that $\Gamma_{v} \vdash \phi \vee \psi$ since $\phi \vee \psi \in \Gamma_{v}$. Suppose $v(\chi)=\mathbf{F} . \quad \Gamma_{v} \nvdash \chi$, so from the fact that CD preserves correctness on $V, \Gamma_{v}, \phi \nvdash \chi$ or $\Gamma_{v}, \psi \nvdash \chi$. If the former, then there is a $v^{\prime} \in V$ such that $v^{\prime}\left(\Gamma_{v}\right)=v^{\prime}(\phi)=\mathbf{T}$ and $v^{\prime}(\chi)=\mathbf{F}$. Since $v^{\prime}\left(\Gamma_{v}\right)=\mathbf{T}, v \leq v^{\prime}$. Since $v^{\prime}(\chi)=\mathbf{F}$ and $v^{\prime}(\phi)=\mathbf{T}$, there is a $v^{\prime}$ extending $v$ such that $v^{\prime}(\chi)=\mathbf{F}$ and $\left[v^{\prime}(\phi)=\mathbf{T}\right.$ or $\left.v^{\prime}(\psi)=\mathbf{T}\right]$. Since $\chi$ was arbitrary, this holds for all such $\chi$. So $C^{\vee}$ holds. The case of $\Gamma_{v}, \psi \nvdash \chi$ is identical. Going the other way, suppose $v(\phi \vee \psi)=\mathbf{F}$. We show there is a formula $\chi$ such that every extension $v^{\prime}$ agreeing with $v$ on $\chi$ has $v^{\prime}(\phi)=v^{\prime}(\psi)=\mathbf{F}$. Take $\phi \vee \psi$ as our $\chi$. Let $v^{\prime} \geq v$ and $v^{\prime}(\phi \vee \psi)=\mathbf{F} . \phi \vdash \phi \vee \psi$ so $v^{\prime}(\phi)=\mathbf{F}$. Likewise we get $v^{\prime}(\psi)=\mathbf{F}$ from $\psi \vdash \phi \vee \psi$. So every $v^{\prime}$ extending $v$ where $\phi \vee \psi$ is false makes both $\phi$ and $\psi$ false.

$C^{\vee}$ expresses that a disjunctive formula is true at $v \in V$ if for every formula false at $v$, one of the two disjuncts is true at some extension of $v$ which agrees with $v$ on the falsity of that formula. Though this condition characterizes the general models which respect $R^{\vee}$, it has a number of rather odd features as I will now demonstrate.

A condition $C^{\#}$ is categorical if, given a set of atomic valuations $U$, there is a unique set of valuations $V$ obeying $C^{\#}$ which agrees with $U$ on $A t . C^{\urcorner}$and $C^{\rightarrow}$ are categorical. $C^{\vee}$ is not categorical. Using $w \leftarrow v$ to indicate $w \leq v$ and writing the atoms true in a valuation next to it in parentheses:

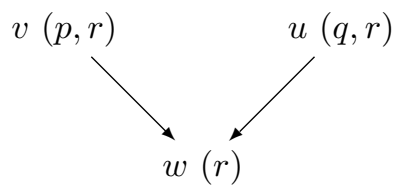

$w(p \vee q)$ may be $\mathbf{T}$ since if it is $\mathbf{T}$, then it can be established by a routine induction on formulas of $\mathfrak{L}_{\vee}$ that for any formula assigned $\mathbf{F}$ by $w$, either $v$ or $u$ assigns that formula $\mathbf{F}$ as well. So for every formula $\rho$ such that $w(\rho)=\mathbf{F}$, there is a $w^{\prime} \geq w$ such that $w^{\prime}(\rho)=\mathbf{F}$ and $w^{\prime}(p)=\mathbf{T}$ or $w^{\prime}(q)=\mathbf{T}$. Furthermore, it is easy to check that in such an extension $w \leq v, u$ and $v, u$ are incomparable. ${ }^{17}$ If, on the other hand, we set $w(p \vee q)$ to $\mathbf{F}$ then there is a formula, $p \vee q$,

${ }^{17}$ For a precise extension, note that if $p \vee q$ is $\mathbf{T}$ at $w$, so too must be $(p \vee s) \vee q$ since 
where the only extension which agrees with $w$ on that formula is $w$ itself where neither $p$ nor $q$ is $\mathbf{T}$. The rest of the atomic model can be fleshed out so that it obeys $C^{\vee}{ }^{18}$ There are thus two extensions of this submodel obeying $C^{\vee}$ which disagree on $w(p \vee q)$ without disturbing $\leq$.

$C^{\vee}$ has another unfortunate property. Given a formula $\phi$, let $S(\phi)$ be the set of strict subformulas of $\phi$. Reverting to our more complicated definition of a model where a model $A$ is a pair $\left\langle W, V^{A}\right\rangle$, call two models $A, B$ agreeing on $W$ $S(\phi)$ - equivalent if and only if for every $w \in W$ and every $\psi \in S(\phi), V_{w}^{A}(\psi)=$ $V_{w}^{B}(\psi)$. Call a condition $C^{\#}$ compositional if, for every formula $\phi$ of $\mathfrak{L}_{\#}$ and every pair $A, B$ of $S(\phi)-$ equivalent models satisfying $C, V_{w}^{A}(\phi)=V_{w}^{B}(\phi)$. It is easy to see that $C^{\rightarrow}$ is not compositional. Consider the following two atomic submodels $A$ and $B$ (all atoms not listed are $\mathbf{F}$ at the relevant valuations).

$$
\begin{array}{cc}
v(r) & u(p, s) \\
v(r) \longleftarrow & u(p, r, s)
\end{array}
$$

Since $\rightarrow$ is categorical, each atomic submodel extends uniquely to a general model. The models are $S(p \rightarrow q)$ - equivalent, but $V_{v}^{A}(p \rightarrow q)=\mathbf{T}$ while $V_{v}^{B}(p \rightarrow q)=\mathbf{F}$. We can thus modify the value of $p \rightarrow q$ by modifying $\leq$ and we can do that by changing the value of atoms which are not subformulas of $p \rightarrow q$. So $C^{\rightarrow}$ is not compositional. However, it obeys a slightly weaker property. Call a condition $C^{\#}$ weakly compositional if, for every formula $\phi$ of $\mathfrak{L}_{\#}$ and every pair $A, B$ of $S(\phi)$-equivalent models satisfying $C$, if $\leq_{A}=\leq_{B}$, then $V_{w}^{A}(\phi)=V_{w}^{B}(\phi)$. It is easily verified that both $C^{\urcorner}$and $C^{\rightarrow}$ are weakly compositional. Unfortunately, $C^{\vee}$ is not.

$p f$. Let $V=\{v, u, w\}$ where $v(p)=v(r)=\mathbf{T}, u(q)=u(r)=\mathbf{T}$, and $w(r)=\mathbf{T}$. All other atomic assignments are to $F$. Then we can flesh out the model so that $w \leq v$ and $w \leq u$ and $u$ and $v$ are incomparable. Our first submodel $(\mathrm{A})$ is familiar from above.

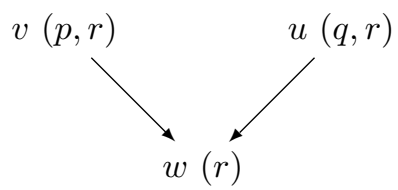

As discussed above, $p \vee q$ may be $\mathbf{T}$ or $\mathbf{F}$ at $w$. Flesh out the model letting $V_{w}^{A}(p \vee q)=\mathbf{T}$. Now, for our second submodel (B), let $V$ be

$(p \vee q) \vee s \vdash \vee(p \vee s) \vee q$ is derivable. So let $V_{w}(\phi)=\mathbf{T}$ if and only if either $r$ is a subformula of $\phi$ or both $p$ and $q$ are. Let $V_{v}(\phi)=T$ if and only if either $p$ or $r$ is subformula. Similarly for $V_{u}$, substituting $q$ for $p$.

${ }^{18}$ Let $V$ be as in fn. 17, changing only $V_{w}$. Let $V_{w}(\phi)=\mathbf{T}$ if and only if $r$ is a subformula of $\phi$. Verification that this and the prior extension obey $C^{\vee}$ is tedious and left to the interested reader. 
as before except to let $w(r)=\mathbf{F}$.

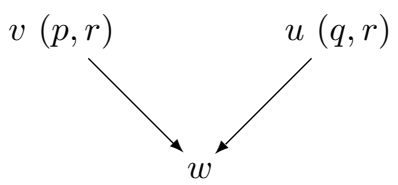

As before, we can flesh this out so that $w \leq u, w \leq v$ and $u$ is incomparable with $v$. However, $V_{w}^{B}(p \vee q)=\mathbf{F}$, since the only extension of $w$ which agrees with $w$ on the truth value of $r$ is $w$. So, the above truth condition collapses into $v(p \vee q)=\mathbf{T}$ iff $v(p)=\mathbf{T}$ or $v(q)=\mathbf{T}$ (the classical truth condition). ${ }^{19}$ Hence, $w(p \vee q)=\mathbf{F}$. Our two models are $S(p \vee q)$ - equivalent and $\leq_{A}=\leq_{B}$, so $C^{\vee}$ is not weakly-compositional.

To properly assess the worrisomeness of these results, we should think about what characterizing $\mathfrak{V}_{R^{\vee}}$ by means of $C^{\vee}$ tells us about the meaning of $\vee$. Even if we presume that a condition defined in terms of the value of formulas on valuations in a general model gives structural constraints on the meaning of a connective, we will not yet have given an account of its meaning until we say what valuations and $\mathbf{T}, \mathbf{F}$ represent. Consider the typical intuitionistic interpretation: take a set of valuations $V$ as consisting of a number of stages of verification (traditionally thought of as verification by an idealized mathematician) ordered by $\leq$, and interpret $v(\phi)=\mathbf{T}$ as indicating that $\phi$ has been verified by stage $v .^{20}$ Thus interpreted, our condition for $\rightarrow$ says that a conditional holds at a verification stage just in case every succeeding verification stage which verifies the antecedent also verifies the consequent. That is, we have verified enough at $v$ that further verification of the antecedent at $u$ extending $v$ directly yields verification of the consequent. This is such a natural interpretation of intuitionistic logic and so familiar that we ought to expect that the natural deduction rules express reasonable meanings for the intuitionistic connectives against it.

The two unpleasant features of $C^{\vee}$ just demonstrated can now be discussed. What the non-categoricity of $C^{\vee}$ shows is that $R^{\vee}$ is insufficient to tell us whether or not verification of one or other disjunct at every succeeding point of a valuation $v$ means that the disjunction counts as verified at $v$. Such a failure is disappointing, but it is by no means devastating. $C^{\vee}$ amounts to a partial constraint on the meaning of $\mathrm{V}$, one to be perhaps filled in in some more direct fashion. The situation here is analogous to that of the failure of the classical deductibility relation to express the boolean interpretation of the connectives. We have the option here as there to accept this partial account of the meaning of the connectives as that which the rules tell us or to search for some modification

\footnotetext{
${ }^{19}$ This generalizes as follows: For any $V$ and any $v \in V$, if there is a formula $\phi$ such that $\neg \exists v^{\prime}\left[v^{\prime} \neq v \leq v^{\prime}\right.$ and $\left.v^{\prime}(\phi)=\mathbf{F}\right]$, then $C^{\vee}$ collapses to the classical truth condition for $\vee$ at $v$.

${ }^{20} \mathrm{~A}$ fuller treatment of this sort of interpretation can be found in Kripke (1965) and Grzegorczyk (1964).
} 
which removes the unintended models.

The failure of weak compositionality is more problematic. What the failure of weak compositionality tells us is that whether or not $p \vee q$ counts as verified at a verification stage $v$ may depend on whether a logically independent atomic sentence $r$ is verified at $v$. But how could the behavior of $r$ at $v$ tell us anything about the verification of $p \vee q$ at $v$ ? Moreover, since $r$ was perfectly general, in order to be confident at a stage $v$ that $p \vee q$ holds at $v$ when $v(p)=v(q)=\mathbf{F}$, it is insufficient to know that (a) we will verify either $p$ or $q$ no matter how we go on to verify, but also (b) that every other sentence of the language currently unverified at this stage will continue to be unverified on some way of continuing to verify. This situation gets worse, of course, for embedded disjunctions.

The upshot is that, sometimes, in order to know that a sentence containing a disjunction holds at a stage, we need to know how every sentence of the language not yet verified - including logically independent sentences - will behave at successive verification stages. This seems entirely too high a bar and, moreover, fits rather badly with both the intuitive intuitionistic interpretation of general models and our basic intuitions about connective meaning. Any intuitionistic interpretation of valuations will be saddled with explaining how the value of an atomic sentence at one valuation can affect the value of a complex formula of which it is not a subformula at another. Anyone further accepting this interpretation of disjunction is further saddled with explaining how the value of an atomic sentence at one valuation can affect the value of a complex formula of which it is not a subformula at another when this does not affect the structure of successive verification stages. It is desirable for connectives to be compositional, but it is mandatory, it would seem, for them to be weakly compositional. The second property thus demands that we augment the constraints on our account of connective meaning.

It follows from 1 and 2 above that no additional rule for $\vee$ which does not extend the intuitionistic deducibility relation will exclude the problematic general models since any rule which does not extend this deducibility relation is already derivable from $R^{\vee} \neg, \rightarrow, \&$. For example, we might be tempted to use the impure rule DS in place of CD to characterize $\vee$ :

$$
\text { (DS) } \frac{\Gamma \vdash \phi \vee \psi \quad \Gamma \vdash \neg \phi}{\Gamma \vdash \psi}
$$

Since our problematic models respect both $R^{\vee}$ and $\left.R\right\urcorner$ and since (DS) is derivable from these, our problematic models respect (DS) as well. So we must do something more direct to remove the problematic models. For the intuitionist, it is not clear what this constraint could be. If we are to maintain a modest inferentialist position, we need to adopt constraints removing such models without moving away from a plausible interpretation of general models all the while maintaining that the rules do the majority, if not quite all, of the work in 
specifying the meaning of each connective. What such a constraint would be is opaque to this author.

For the classical logician the constraint mentioned in our earlier discussion of the classical modest inferentialist might work. Following Garson and Rumfitt, we can take a set of valuations $V$ as consisting of possibilities-partial specification of possible worlds. We can then take $v(\phi)=\mathbf{T}$ as $\phi$ is part of the specification $v, v(\phi)=\mathbf{F}$ as $\phi$ is not part of the specification $v$, and $v \leq u$ as $u$ is a fuller specification than $v$. The conditions given by this account are fairly natural: $v(p \rightarrow q)=\mathbf{T}$ amounts to: $q$ is part of any specification which includes everything $v$ includes and $p$. Then we can eliminate the above models by enforcing the condition:

$$
(\mathrm{LF}) v(\phi)=\mathbf{F} \Rightarrow \exists v^{\prime}\left[v \leq v^{\prime} \wedge \forall v^{\prime \prime}\left(v^{\prime} \leq v^{\prime \prime} \Rightarrow v^{\prime \prime}(\phi)=\mathbf{F}\right)\right]^{21}
$$

When we narrow our class of models satisfying the intuitionistic natural deduction rules to those which meet LF, double negation elimination and Peirce's law are validated. The trouble with this approach is motivating LF as part of our intuitive account of possibilities. ${ }^{22}$ Consider a case where we have two specifications. One a full specification of a possible world, the other a subspecification where we remove a single piece of information and made the rest of the necessary changes. It is hard to see why this does not count as an intuitively acceptable set of specifications. ${ }^{23}$ More work would have to be done to motivate

\footnotetext{
${ }^{21}$ Garson (forthcoming) suggests that we might replace LF with a condition pLF quantifying over only atomic sentences. Unfortunately, while this restores weak compositionality, it is insufficient for categoricity - pLF holds in our example demonstrating failure of categoricity. Further, expanding our language and noting that our model respects $R\urcorner$, we can extend the atomic model so $\neg \neg(p \vee q)$ is $\mathbf{T}$ on the same valuation where $p \vee q$ is $\mathbf{F}$. The upshot is that pLF is not a strong enough side condition to validate double negation elimination or to enforce categoricity.

${ }^{22}$ There are similar problems for other intuitive interpretations. Consider a tensed interpretation of classically admissible general models in which valuations represent particular moments of time. Now suppose some omnipotent malicious being is eternally toying with destroying the world in an instant. Let $\phi$ express that this being has decided to do so. Here's a simple (and familiar!) model representing one way this might go:

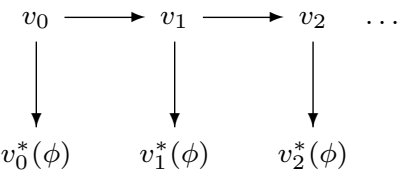

where each terminal *-ed valuation represents the last moment of time since $\phi$ is true there. LF tells us such a general model is not admissible and, thus, that such a situation is not conceptually possible. But logic should not assuage such worries. Similar worries arise for an epistemic interpretation of the class of general models respecting the natural deduction rules for classical logic. (In personal correspondence, Garson has suggested a more plausible tensed interpretation.)

${ }^{23}$ More plausible constraints on the background semantic framework are possible, but they do not remove our troublesome models. For example, we might require that a sentence not included in a specification would either be $\mathbf{T}$ at some fuller specification or $\mathbf{F}$ at some fuller specification and every fuller specification thereof. This captures a sense in which specifications could always be made more definite. But our troublesome models remain.
} 
this approach to our problem.

On the other hand, if we take LF to be part of the meaning of classical negation as expressed by the natural deduction rules, not as part of our prelogical semantic assumptions, then we are faced with another problem. Classical negation is proof-theoretically conservative over disjunction - unlike the case of the conditional and classical negation, every sentence of $\mathfrak{L}_{\vee}$ provable on the basis of $R^{\vee} \neg$ and double negation elimination was already provable on the basis of $R^{\vee}{ }^{24}$ Yet the condition expressed by double negation elimination changes the meaning of $\vee$ by eliminating atomic models, yielding a categorical refinement of the meaning. It seems out of the spirit of modest inferentialism to allow failures of conservativeness than cannot, like Peirce's law, be traced to failures of prooftheoretic conservativeness. This novel form of non-conservativeness should be addressed by classical modest inferentialists.

\section{References}

Carnap, R. (1959). Introduction to Semantics and Formalization of Logic. Harvard University Press.

Garson, J. (2001). Natural Semantics: Why Natural Deduction is Intuitionistic. Theoria, 67(2):114-139.

Garson, J. (Forthcoming). What Logic Means: From Proof to Model-Theoretic Semantics.

Grzegorczyk, A. (1964). A Philosophically Plausible Formal Interpretation of Intuitionistic Logic. Indagationes Mathematicae, 26(5):596-601.

Humberstone, L. (1981). From Worlds to Possibilities. Journal of Philosophical Logic, 10(2):313-339.

Humberstone, L. (1996). Valuational Semantics of Rule Derivability. Journal of Philosophical Logic, 25(5):451-461.

Kripke, S. A. (1965). Semantical analysis of intuitionistic logic. In Crossley, J. and Dummett, M. A., editors, Formal Systems and Recursive Functions. 92-130. North-Holland, Amsterdam.

Peacocke, C. (1976). What is a Logical Constant? The Journal of Philosophy, 73(9):221-240.

Prawitz, D. (1985). Remarks on Some Approaches to the Concept of Logical Consequence. Synthese, 62(2):153-171.

Restall, G. (2005). Multiple Conclusions. In Logic, Methodology and Philosophy of Science: Proceedings of the Twelfth International Congress. 189-205. Kings College Publications.

${ }^{24}$ See (Steinberger, 2011, 625). 
Rumfitt, I. (2012). On A Neglected Path to Intuitionism. Topoi, 31(1):101-109.

Shoesmith, D. and Smiley, T. (1978). Multiple-Conclusion Logic. CUP Archive.

Smiley, T. (1996). Rejection. Analysis, 56(1):1-9.

Steinberger, F. (2011). What Harmony Could and Could Not Be. Australasian Journal of Philosophy, 89(4):617-639. 